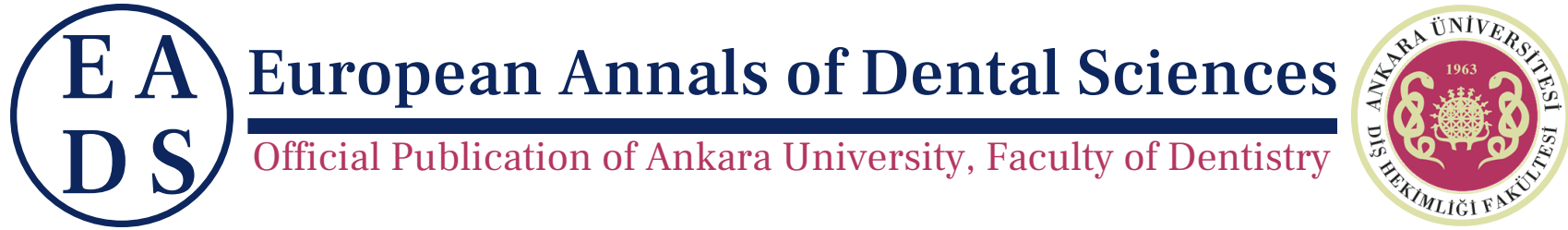

EADS, 2021, 48 (3), 95-100

\title{
Comparison of Diode Laser and Conventional Method in Treatment of Gingival Melanin Hyperpigmentation
}

\author{
Revan Birke Koca-Ünsal ${ }^{1}$,* , Gökhan Kasnak ${ }^{2}$ and Erhan Frratlı $\odot 3$ \\ ${ }^{1}$ Department of Periodontology, Faculty of Dentistry, University of Kyrenia, Kyrenia, Cyprus and ${ }^{2}$ Department of \\ Periodontology, Faculty of Dentistry, Istanbul Kent University, Istanbul, Turkey and ${ }^{3}$ Department of Periodontology, \\ Faculty of Dentistry, Istanbul University, Istanbul, Turkey \\ *Corresponding Author; revanbirke.koca@kyrenia.edu.tr
}

\begin{abstract}
Purpose: The goal of this study is to compare the scalpel and diode laser methods for treating gingival hyperpigmentation in terms of postoperative discomfort and wound healing.

Materials \& Methods: Sixteen systemically healthy individuals diagnosed with light or moderate gingival hyperpigmentation were enrolled for this study. Patients were randomly assigned to one of two treatment groups: scalpel or laser. Dummett's oral pigmentation index was recorded at baseline. Early wound healing and post-operative discomfort were evaluated on the 7th day by using the VAS form. Comparisons between the groups were tested using the Mann-Whitney U test and P-value $<0.05$ was considered significant.

Results: Total epithelization was observed in the laser group, whereas the epithelization was incomplete in the scalpel group at the end of the observation period. The pain perception on the first two days after the surgery was significantly higher in the scalpel group than in the laser group. ( $\mathrm{p}=0,002$ and $\mathrm{p}=0,038$, respectively). No significant differences were found between the fourth- and seventh-day when surgical techniques were compared regarding pain perception $(p>0,05)$. Similarly, pain perceptions of female and male individuals showed no significant difference ( $p>0,05)$.

Conclusion: In the treatment of gingival hyperpigmentation, both surgical techniques were clinically successful. Although the treatment process was long, according to the results of our study, the laser technique is superior to the scalpel method in terms of patient comfort. The choice of the method may vary depending on the available equipment and the clinician's preference or request of the patient.
\end{abstract}

Key words: diode laser; gingival hyperpigmentation; postoperative pain; scalpel; VAS

\section{Introduction}

Pathologically or physiologically, "hyperpigmentation" refers to an excess of melanin pigment in tissue, whereas gingival hyperpigmentation is the excessive accumulation of the melanin pigment at the basal and suprabasal layers of the oral epithelium ${ }^{1}$. There is no direct correlation between the etiology of melanin pigmentation and race, age, or gender; however, drugs, heavy metals, and hormonal disorders might be associated with hyperpigmentation ${ }^{2,3}$ In addition, smoking might provoke gingival pigmentation and the severity of the pigmentation can be related to the number of cigarettes smoked per dayhed ${ }^{4,5}$. According to literature, gingival discolorations might clinically show a diffuse pattern in the form of a smooth or scattered patch in dark colors such as brown/purple ${ }^{6}$. Although typical hyperpigmentation of the gingiva does not generally pose a medical risk, it might be an esthetic problem for people with high smile lines.

From the past to the present, various treatment methods have been applied for gingival depigmentation such as scalpel technique $^{4}$, bur abrasion, cryosurgery ${ }^{7,8}$, electrosurgery ${ }^{9}$, pharmaceutical $^{10}$ and mucogingival ${ }^{11}$ surgical procedures (free gingival graft or dermal matrix allograft) and laser applications ${ }^{12,13}$. Many aspects should be considered by clinicians when deciding on treatment approaches, including the prevalence, intensity, and severity of gingival hyperpigmentation. Furthermore, the surgical skills/experience of the clinician and the availability of equipment are factors that can directly impact the selection of the treatment protocol. The scalpel method is the most preferred method by clinicians in treating gingival hyperpigmentation, as it does not require any special equipment, is easy to apply, and delivers prospering results. In this approach, the scalpel is held parallel to the gingival surface and scraped the epithelial layer of the hyperpigmented gin- 
giva, including the basal layer where the melanocytes are located. Although the scalping method has many advantages, postoperative bleeding and patient discomfort are among the main disadvantages of the technique.

In recent years, lasers have been used in gingival surgery as well as other areas of dentistry, and they are frequently preferred by clinicians to treat gingival hyperpigmentation as an alternative to scalping. Although lasers are expensive technologies, one of the reasons for this preference might be the comfort afforded to clinicians by bleeding control in laser use. Another cause could be improved patient comfort as a result of reduced pressure perception during the procedure. The most commonly used lasers in melanin depigmentation are Er: YAG, Nd: YAG, $\mathrm{CO} 2$, Argon, and the diode laser, which is the most preferred one by clinicians.

We aim to evaluate the postoperative pain with The Visual Analog Scale (VAS) and clinical appearance of wound healing by comparing scalping and diode laser methods to treat gingival hyperpigmentation.

\section{Materials and Methods}

\section{Study Design}

Twenty individuals who were referred to Istanbul University Faculty of Dentistry Department of Periodontology for esthetic complaints due to gingival pigmentation. After obtaining informed consent, all individuals were assessed by the same practitioner (G.K). The Dummett-Gupta oral pigmentation index (DOPI) ${ }^{1 / 4}$ was used for the evaluation of the pigmentation and a full periodontal examination, including plaque index $(\mathrm{PI})^{15}$, gingival index $(\mathrm{GI})^{16}$, clinical attachment level (CAL), and probing pocket depth (PPD) measurements at six sites of each teeth (UNC-15, Hu-Friedy, Chicago, IL, USA) was carried out to determine the clinical periodontal status. Digital orthopantomographs were taken from all individuals (KODAK 9000 3D, Carestream Dental LLC, Atlanta, GA, USA) (Dental Imaging Software CS 3D, Carestream Dental LLC, Atlanta, GA, USA) to assess the alveolar bone level. Four smoker individuals with heavy pigmentation were excluded from the study group. Sixteen (eight females and eight males, in the age range of 23 to 41) non-smoker and systemically healthy individuals who had light or moderate gingival hyperpigmentation with no signs of gingival inflammation, no alveolar bone loss, no sites with $\mathrm{PPD}>3 \mathrm{~mm}$ and $\mathrm{CAL}>2$ $\mathrm{mm}$ were identified as periodontally healthy and selected for this study (Table 1). Neither oral hygiene instruction was given, nor initial periodontal treatment was performed on any of the individuals prior to the depigmentation procedure.

\section{Gingival Melanin Index}

According to Dummett Oral Pigmentation Index (DOPI) ${ }^{1 / 4}$, gingival pigmentation classification is as follows:

Score 1: No clinical pigmentation (pink gingiva)

Score 2: Slight clinical pigmentation (slightly brown gingiva)

Score 3: Moderate clinical pigmentation (medium brown or mixed pink and brown gingiva)

Score 4: Severe clinical pigmentation (dark brown or bluish-black gingiva)

The gingival melanin index of the participants before and after depigmentation was determined with the help of this index and the values were recorded.

The individuals were randomly assigned to the scalpel or diode laser group and all of the depigmentation procedures were carried out by the same calibrated practitioner (R.B.K.Ü). At the end of the procedures, all individuals were advised to use chlorhexidine gluconate oral spray ( $0,2 \%$, Klorhex, Drogsan Ltd, Istanbul, Turkey) for a week. Neither nonsteroidal anti-inflammatory analgesics nor antibiotics were prescribed to evaluate the postoperative pain perception definitively. In addition, all patients were strictly instructed to notify the clinician if they took any prescription or nonprescription medication during the recovery period. All patients were given a VAS form which included a scale of 1-5 for each day to mark the pain they felt on the 1st, 2nd, 4 th and 7 th day after the procedure (Figure 1). At 1-week follow-up, the wound healing was evaluated clinically, and photographs were taken of depigmented parts.

\section{Depigmentation with Scalpel}

Local infiltration anaesthesia ( $4 \%$ articaine $\mathrm{HCl}$ with 1:100.000 epinephrine; Ultracaine D-S Forte, Sanofi Aventis, Frankfurt, Germany) was administered to the operation site. A15C scalpel (SwannMorton Ltd., Sheffield, UK) was placed parallel to the gingival surface with taken care not to extend the mucogingival line. Gingivoplasty was performed by removing the epithelial tissue in each area with pigmentation starting from the gingival margin until the slight bleeding of connective tissue occurs. During the procedure to prevent excessive trauma, the operation site was irrigated with sterile saline water ( $0.9 \% \mathrm{NaCl}$, Polifarma, Istanbul, Turkey). The operator had observed the scalpel blade's sharpness during the surgery and changed it when the blade's performance was insufficient. Moisturized gauze pads were applied to the sites with bleeding for five minutes. The periodontal dressing was not applied to the area in order not to affect postoperative pain.

\section{Depigmentation with Diode Laser}

Local infiltration anaesthesia (4\% articaine $\mathrm{HCl}$ with 1:100.000 epinephrine; Ultracaine D-S Forte, Sanofi Aventis, Frankfurt, Germany) was administered to the operation site. A diode laser (Doctor Smile, Vicenza, Italy) with $810 \mathrm{~nm}$ wavelength and $1 \mathrm{~W}$ was used for the depigmentation procedure. The laser was set to continuous wave mode and the $320 \mathrm{~nm}$ tip was kept in contact with the pigmented area during the ablation. The fiber optic tip was angulated approximately 45 degrees to the long axis of the teeth and applied from mucogingival junction to the direction of the gingival margin with paint brush strokes. Moisturized gauze pads were applied to clean the carbonized tissue remnants from the tip and moisturize the surgical area.

\section{Statistical Analysis}

The data were recorded in a Microsoft Excel sheet and imported to a statistical computing software (SPSS v.27 for Windows, IBM Inc., Chicago, USA) for further analyses. The differences of the periodontal indices and DOPI scores were compared regarding gender and operation technique by using the Student t-test. The obtained data were not distributed normally, and therefore comparisons between the groups were tested using the Mann-Whitney U test. Results were represented as mean \pm standard deviation $(\mathrm{SD})$ and $\mathrm{P}$-value < 0.05 was considered significant.

\section{Results}

The demographic data, the periodontal index scores, and DOPI values of the individuals were given in Table 1. Clinical parameters and age showed no statistically significant difference in terms of gender and operation technique $(p>0,05)$. At the end of the seventh day, the individuals in the scalpel group showed total epithelialization, whilst the individuals in the laser group showed incomplete epithelialization. Nonetheless, no impaired wound healing was reported by any of the individuals or observed by the practitioner (Figure 2). At the end of the follow-up period, all patients in the scalpel group 




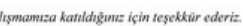

Figure 1. VAS (Visual Analogue Scale) Form

were extremely satisfied with the esthetic results.

Individuals in the scalpel group significantly declared higher pain perception in comparison to the diode laser group on the first and second days after the surgery $(p=0,002$ and $p=0,038$, respectively). No significant differences were found between the groups on the fourth- and seventh-day regarding pain perception ( $p$ > 0,05 ) (Figure 3). Also, the pain perception in female and male individuals regarding the depigmentation technique was compared, and no significant difference was observed in any comparisons $(\mathrm{p}>$ 0,05) (Figure 4).

\section{Discussion}

Oral melanin hyperpigmentation is an endogenous or exogenous condition characterized by an excess of melanin pigment accumulation due to various of local and systemic factors. It can be found in individuals of all ages, genders, and races. Exogenous pigmentations occurs when the gingiva is exposed to foreign-body and stained. However, the occurrence of endogenous pigmentation can be physiological, pathological, or metastatic ${ }^{17}$. The differential diagnosis of oral melanin pigmentation is critical since certain benign lesions might be malignant with a poor prognosis ${ }^{18}$.

Although the physiological melanin pigmentation does not cause a medical problem, it might be an esthetic concern for many individuals, especially those with a high smile line. Gingival hyperpigmentation can be treated using a variety of techniques in today's esthetic dental practices. In the present study, individuals with light or moderate gingival hyperpigmentation were treated with either scalpel or diode laser. The post-operative discomfort and early wound healing of the individuals regarding the surgical tech-

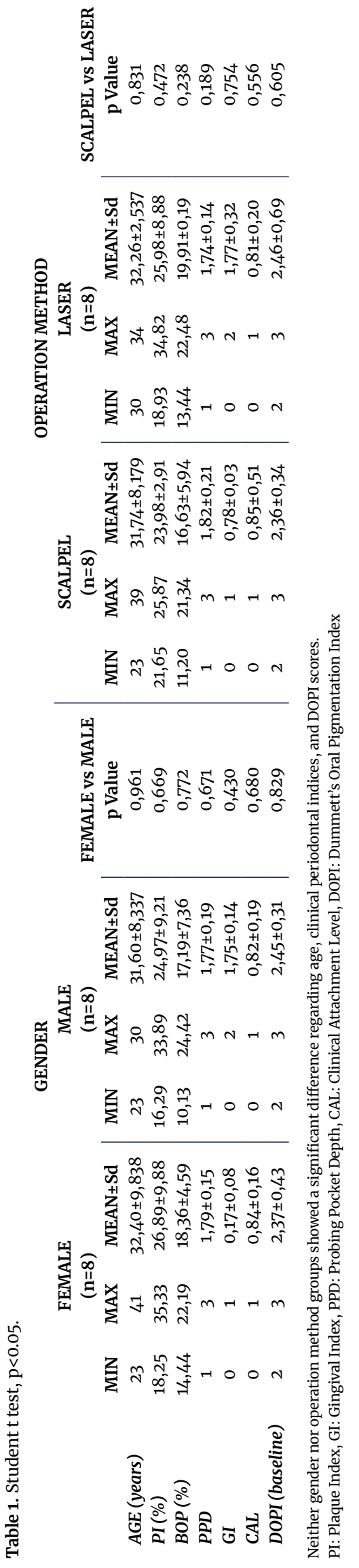




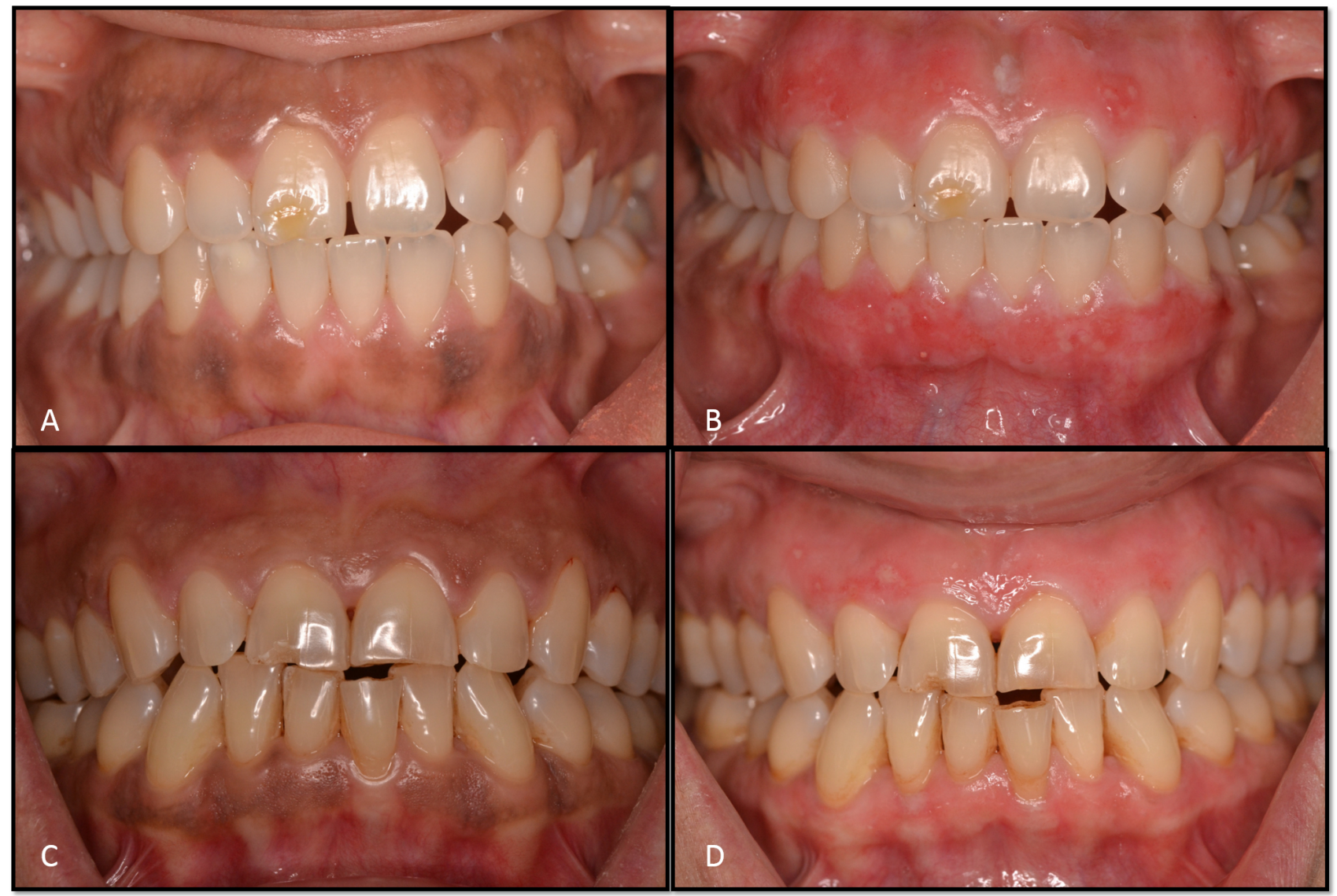

Figure 2. Intraoral view of the study groups (A: Laser group, baseline; B: Laser group, postoperative 7th day; C: Scalpel group, baseline; D: Scalpel group, postoperative 7th day)

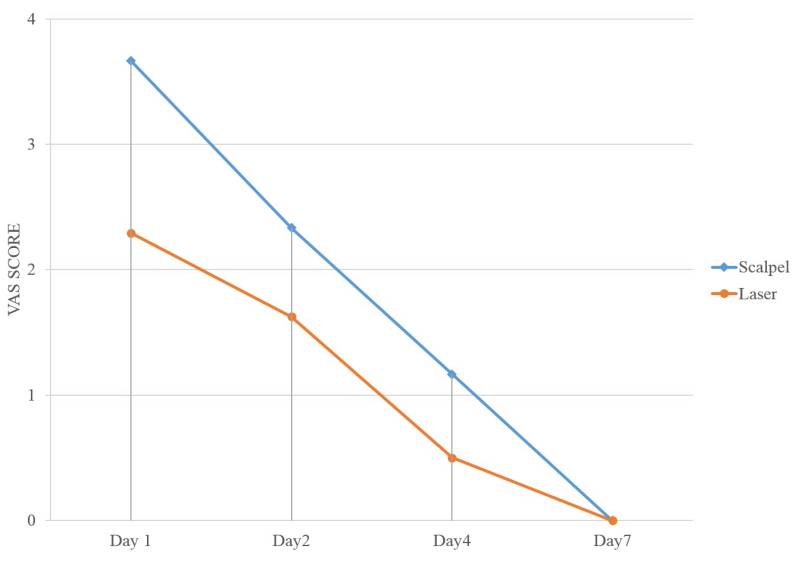

Figure 3. The scalpel and diode laser techniques were compared. The VAS score was significantly high in the scalpel group on the first and second days after the operation. No significant difference was observed $(* \mathrm{p}=0,002$ and $* * \mathrm{p}=0,038)$ between the groups on the fourth and seventh days $(p>0,05)$.

nique were compared. The patients' pain perception was evaluated according to the Visual Analog Scale (VAS), which is a well-known and widely used tool to validate the subjective experience of the pain intensity Besides, the absence of a language and ease of application are the other advantages of this method ${ }^{19}$. The pain scale is scored from "no pain" to "unbearable pain," and the patient is asked to mark the number that best describes his or her discomfort on the designated days. The average of the numbers marked by the patients in each group is calculated and the pain level for that group is found. In order to include illiterate patients in our study, visual elements were assigned for each score, which was equivalent to the

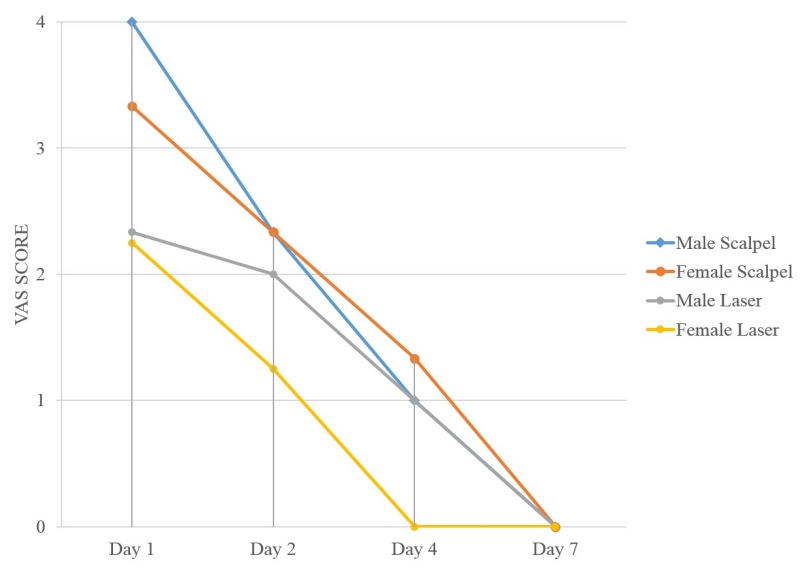

Figure 4. The VAS score of the female individuals in the scalpel group and diode laser group were compared. Similarly, the VAS score of the male individuals between the scalpel and diode laser groups was compared. Also, the comparison between male and female individuals was carried out for each surgical technique separately. No significant difference was detected in any comparisons ( $p>0.05)$.

pain level.

According to our findings, there was no significant difference between the VAS scores of the scalpel group and the laser group. However, VAS scores of the laser group were significantly lower than the scalpel group when scores for the first two days were compared. One explanation for this might be a rapid formation of the coagulum, which covers the nerve endings and alleviates the sense of pain in laser-assisted depigmentation ${ }^{20}$. Considering the laser and scalpel groups separately for males and females, although the VAS in the scalpel group was slightly higher than the laser group 
in both groups, there was no significant difference within or between groups. In this study, the reason for grouping depigmentation methods with gender was to measure the pain levels of males or females according to different methods. However, the mean values showed that it was not necessary to select the treatment method by gender ${ }^{21}$.

Although there are many indices in the literature which classify the severity and prevalence of oral pigmentation, the Dummett Oral Pigmentation Index (DOPI) is still the most widely used in studies and considered the gold standard ${ }^{14}$. The DOPI values of the laser and the scalpel groups were similar at baseline, there was no significant difference between them. Similarly, the comparison of VAS score between male and female individuals was carried out for each surgical technique separately. No significant difference was detected in any comparisons.

In the scalpel group, the chair-time was relatively shorter; however, the intraoperative hemorrhage and postoperative pain were significantly higher. It was observed that epithelization continued clinically even on the 7th postoperative day. The gingival depigmentation with surgical blades is a straightforward, practical, and less expensive treatment option. Still, it requires greater clinical experience due to the possibility of alveolar bone exposure in individuals with a thin gingival phenotype. The main disadvantages of this technique, including intraoperative bleeding, postoperative pain, and edema, have been addressed by several studies 4,22,23.

The diode laser has continuous and pulsative modes in the wavelength range of $800-980 \mathrm{~nm}^{9}$ and causes minimal harm to the periosteum and alveolar bone during the operation and ensures proper removal of the epithelial layer ${ }^{24}$. Since it does not interact with hard tissue, it is indicated for soft tissue procedures such as gingiva. As the fiber tip causes heat accumulation during use, thermal effects are observed on the tissue surface and a thick coagulation layer forms and provides homeostasis ${ }^{25}$. In our study, during the treatment of gingival hyperpigmentation with a diode laser, we almost did not observe bleeding, albeit the operation time was longer. In addition to this, the use of diode lasers in the gingival depigmentation procedures provides advantages over the other laser types due to their compact structure, relatively low costs, and ease of use ${ }^{26}$.

Several studies in the literature reported that the wound heals faster when the depigmentation is performed with a scalpel $^{11,13,26,27}$. Consistent with the present literature, faster epithelization was observed in the scalpel group compared to the laser group in our study. Although wound healing continued in the 1stweek follow-up, early post-operative epithelialization was better in the scalpel group. According to some researchers, this was the result of thermal damage to tissues by the diode laser ${ }^{28}$. Nevertheless, reduced postoperative pain is the remarkable benefit of the diode laser to the scalpel in early phase wound healing ${ }^{29}$.

One limitation of the study is the relationship between postoperative pain and the scalpel's sharpness could not be interpreted because the number of scalpels used was not noted. Our study aimed to evaluate the effect of the two surgical techniques on early wound healing as the follow-up period was up to the 7th post-operative day. Therefore, long-term studies should be planned to assess the effectiveness of the surgical techniques on the recurrence of pigmentation. Moreover, the effect of smoking on oral melanin hyperpigmentation is undeniably high and the rate of recurrence after depigmentation varies depending on the frequency and amount of smoking ${ }^{30}$. The inclusion of smokers in future studies is important in terms of evaluating both wound healing rate and recurrences.

\section{Conclusion}

Successful clinical results can be obtained with both scalpel and diode laser in the treatment of oral melanin hyperpigmentation. However, intraoperative homeostasis and relatively less postopera- tive pain reveal the superiority of diode lasers to the scalpel. Besides, there are many safe and effective techniques for depigmentation, such as cryosurgery or different types of lasers. The choice of the method may vary depending on the available equipment and preference of the patient and the clinician.

\section{Author Contributions}

RBKÜ and GK designed and directed the project; RBK undertook the treatments and RBKÜ and EF followed up of the patient. GK contributed to the analysis of the study, RBKÜ, GK and EF contributed to the interpretation of the results. All authors provided critical feedback and helped to shape the manuscript.

\section{Conflict of Interest}

The authors declare that they have no affiliations with or involvement in any organization or entity with any financial interest or non-financial interest in the subject matter or materials. This research did not receive any specific grant from funding agencies in the public, commercial, or not-for-profit sectors.

\section{Authors' ORCID(s)}

$\begin{array}{ll}\text { R.B.K.U. } & 0000-0003-1540-983 X \\ \text { G.K. } & 0000-0002-8953-6834 \\ \text { E.F. } & 0000-0002-4154-6929\end{array}$

\section{References}

1. Cicek Y, Ertas U. The normal and pathological pigmentation of oral mucous membrane: a review. J Contemp Dent Pract. 2003;4(3):76-86.

2. Lenane P, Powell FC. Oral pigmentation. J Eur Acad Dermatol Venereol. 2000;14(6):448-65. doi:10.1046/j.14683083.2000.00143.x.

3. Sarswathi TR, Kumar SN, Kavitha KM. Oral melanin pigmentation in smoked and smokeless tobacco users in India. Clinicopathological study. Indian J Dent Res. 2003;14(2):101-6.

4. Alhabashneh R, Darawi O, Khader YS, Ashour L. Gingival depigmentation using Er:YAG laser and scalpel technique: A six-month prospective clinical study. Quintessence Int. 2018;49(2):113-122. doi:10.3290/j.qi.a39267.

5. Hedin CA, Axell T. Oral melanin pigmentation in 467 Thai and Malaysian people with special emphasis on smoker's melanosis. J Oral Pathol Med. 1991;20(1):8-12. doi:10.1111/j.16000714.1991.tboo879.x.

6. Namdeoraoji Bahadure R, Singh P, Jain E, Khurana H, Badole G. Management of pigmented gingiva in child patient: a new era to the pediatric dentistry. Int J Clin Pediatr Dent. 2013;6(3):197200. doi:10.5005/jp-journals-10005-1218.

7. Rahmati S, Darijani M, Nourelahi M. Comparison of surgical blade and cryosurgery with liquid nitrogen techniques in treatment of physiologic gingival pigmentation: short term results. J Dent (Shiraz). 2014;15(4):161-6.

8. Tal H, Landsberg J, Kozlovsky A. Cryosurgical depigmentation of the gingiva. A case report. J Clin Periodontol. 1987;14(10):6147. doi:10.1111/j.1600-051x.1987.tb01525.x.

9. Chandna S, Kedige SD. Evaluation of pain on use of electrosurgery and diode lasers in the management of gingival hyperpigmentation: A comparative study. J Indian Soc Periodontol. 2015;19(1):49-55. doi:10.4103/0972-124X.145823.

10. Hirschfeld I, Hirschfeld L. Oral pigmentation and a method of 
removing it. Oral Surg Oral Med Oral Pathol. 1951;4(8):1012-6. doi:10.1016/0030-4220(51)90448-3.

11. Murthy MB, Kaur J, Das R. Treatment of gingival hyperpigmentation with rotary abrasive, scalpel, and laser techniques: A case series. J Indian Soc Periodontol. 2012;16(4):614-9. doi:10.4103/0972-124X.106933.

12. Atsawasuwan $P$, Greethong $K$, Nimmanon V. Treatment of gingival hyperpigmentation for esthetic purposes by Nd:YAG laser: report of 4 cases. J Periodontol. 2000;71(2):315-21. doi:10.1902/jop.2000.71.2.315.

13. Bakhshi M, Rahmani S, Rahmani A. Lasers in esthetic treatment of gingival melanin hyperpigmentation: a review article. Lasers Med Sci. 2015;30(8):2195-203. doi:10.1007/s10103-0151797-3.

14. Dummett CO, Barens G. Oromucosal pigmentation: an updated literary review. J Periodontol. 1971;42(11):726-36. doi:10.1902/jop.1971.42.11.726.

15. Silness J, Loe H. Periodontal Disease in Pregnancy. Ii. Correlation between Oral Hygiene and Periodontal Condtion. Acta Odontol Scand. 1964;22:121-35. doi:10.3109/00016356408993968.

16. Loe H, Silness J. Periodontal Disease in Pregnancy. I. Prevalence and Severity. Acta Odontol Scand. 1963;21:533-51. doi:10.3109/00016356309011240.

17. Kauzman A, Pavone M, Blanas N, Bradley G. Pigmented lesions of the oral cavity: review, differential diagnosis, and case presentations. J Can Dent Assoc. 2004;70(10):682-3.

18. Koca RB, Unsal G, Soluk Tekkesin M, Kasnak G, Orhan K, Ozcan I, et al. A review with an additional case: amelanotic malignant melanoma at mandibular gingiva. Int Cancer Conf $\mathrm{J}$. 2020;9(4):175-181. doi:10.1007/s13691-020-00425-3.

19. Wewers ME, Lowe NK. A critical review of visual analogue scales in the measurement of clinical phenomena. Res Nurs Health. 1990;13(4):227-36. doi:10.1002/nur.4770130405.

20. Simsek Kaya G, Yapici Yavuz G, Sumbullu MA, Dayi E. A comparison of diode laser and Er:YAG lasers in the treatment of gingival melanin pigmentation. Oral Surg Oral Med Oral Pathol Oral Radiol. 2012;113(3):293-9. doi:10.1016/j.tripleo.2011.03.005.

21. Grover HS, Dadlani H, Bhardwaj A, Yadav A, Lal S. Evaluation of patient response and recurrence of pigmentation following gingival depigmentation using laser and scalpel technique: A clinical study. J Indian Soc Periodontol. 2014;18(5):586-92. doi:10.4103/0972-124X.142450.

22. Amaral MB, de Avila JM, Abreu MH, Mesquita RA. Diode laser surgery versus scalpel surgery in the treatment of fibrous hyperplasia: a randomized clinical trial. Int J Oral Maxillofac Surg. 2015;44(11):1383-9. doi:10.1016/j.ijom.2015.05.015.

23. Jin JY, Lee SH, Yoon HJ. A comparative study of wound healing following incision with a scalpel, diode laser or Er,Cr:YSGG laser in guinea pig oral mucosa: A histological and immunohistochemical analysis. Acta Odontol Scand. 2010;68(4):232-8. doi:10.3109/00016357.2010.492356.

24. Ortega-Concepcion D, Cano-Duran JA, Pena-Cardelles JF, Paredes-Rodriguez VM, Gonzalez-Serrano J, Lopez-Quiles J. The application of diode laser in the treatment of oral soft tissues lesions. A literature review. J Clin Exp Dent. 2017;9(7):e925-e928. doi:10.4317/jced.53795.

25. Aboujaoude S, Cassia A, Moukarzel C. Diode Laser Versus Scalpel in the Treatment of Hereditary Gingival Fibromatosis in a 6-Year Old Boy. Clin Pract. 2016;6(4):895. doi:10.4081/cp.2016.895.

26. Gupta G. Management of gingival hyperpigmentation by semiconductor diode laser. J Cutan Aesthet Surg. 2011;4(3):208-10. doi:10.4103/0974-2077.91256.

27. Suragimath G, Lohana MH, Varma S. A Split Mouth Randomized Clinical Comparative Study to Evaluate the Efficacy of Gingival Depigmentation Procedure Using Conventional Scalpel Technique or Diode Laser. J Lasers Med Sci. 2016;7(4):227-232. doi:10.15171/jlms.2016.40.

28. Ize-Iyamu IN, Saheeb BD, Edetanlen BE. Comparing the 810nm diode laser with conventional surgery in orthodontic soft tissue procedures. Ghana Med J. 2013;47(3):107-11.

29. Muruppel AM, Pai BSJ, Bhat S, Parker S, Lynch E. LaserAssisted Depigmentation-An Introspection of the Science, Techniques, and Perceptions. Dent J (Basel). 2020;8(3). doi:10.3390/dj8030088.

30. Unsal E, Paksoy C, Soykan E, Elhan AH, Sahin M. Oral melanin pigmentation related to smoking in a Turkish population. Community Dent Oral Epidemiol. 2001;29(4):272-7. doi:10.1034/j.1600-0528.2001.290406.x. 